\title{
Longitudinal sliding of the median nerve during movements of the upper limb
}

\author{
D. L. M C LELLAN AND M. SWASH \\ From the Department of Neurology, Section of Neurological Sciences, \\ The London Hospital, London
}

SYNOPSIS An incidental finding during recordings from the median nerve was that the nerve trunk slides longitudinally in its bed when the limb is moved. The amount of movement has been measured in 19 subjects. The observations are discussed with reference to the pathology of entrapment neuropathies and it is suggested that, where longitudinal movement of a peripheral nerve is restricted, continual trauma results from normal movements of the limb.

When a limb moves, the beds or channels through which peripheral nerves run may shorten or lengthen. The nerve fibres in a peripheral nerve are arranged in a spiral fashion so that some elongation can occur by untwisting without necessarily altering the length of individual fibres (Clarke and Bearn, 1972). In certain positions of a limb a nerve trunk may be free of tension and then lies lax in its bed, allowing slight undulations in its course. Lengthening of the nerve bed will thus lead first to straightening of the nerve, then to stretching (Sunderland, 1968) and untwisting. These adaptive mechanisms allow local changes in the length of a nerve bed, as might occur for example during flexion of the wrist, to be accommodated by the whole nerve. This will necessarily cause the nerve trunk to slide longitudinally in its bed at some distance from the point at which stretch is applied.

While recording afferent discharges in single nerve fibres in the median nerve in man we have observed that such sliding movement commonly occurs (McLellan, 1975). In this paper we describe the amount of sliding that took place and we suggest that this mechanical movement is an important factor in entrapment neuropathy.

(Accepted 9 February 1976.)

\section{METHODS}

Observations were made during the recording o action potentials in single afferent nerve fibres in the median nerve of 15 subjects whose ages varied from 19 to 72 (mean 48 ) years. The observations were made with the patient lying supine on की couch, the arm being abducted at about $45^{\circ}$ from. $\overrightarrow{0}$ the trunk. A tungsten needle electrode with shaft $0.3 \mathrm{~mm}$ in diameter, tapering at its tip to $5 \mu \mathrm{m}$, was inserted through the skin of the upper arm into the trunk of the median nerve at a point half way between the elbow and the shoulder. No anaesthetic was used. The electrode was insulated except at the tip to give an impedance of 150 kohms at $1000 \mathrm{~Hz}$, as described by Vallbo and Hagbarth (1968). In four other subjects (aged between 22 and 41 years) similar observations were made with an electrode inserted into the median nerve at the wrist.

The subject usually knew when the electrode had entered the nerve because of a shower of paraesthesiae in its cutaneous distribution. As soon as the electrode stopped moving these paraesthesiae subsided. The location of the electrode was confirmed by recording action potentials from single sensory nerve fibres in the manner described by Vallbo and Hagbarth (1968).

Active and passive movements of the limb were then performed. Such movements produced angulation of the electrode, indicating that its tip had $N$ moved relative to its site of entry through the $\mathrm{W}$ skin. This movement was always in the long axis of the nerve (Fig. 1). The movement was always 


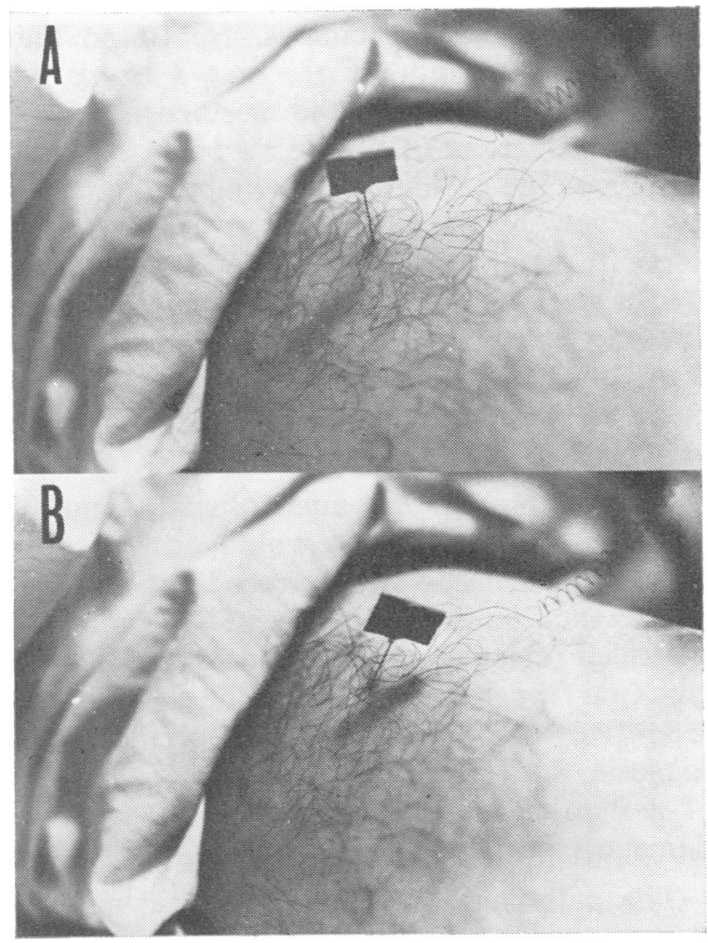

FIG. 1 Photographs of the upper arm to show angulation of the electrode. The shoulder is off the picture to the left, the elbow off right. The tip of the electrode is in the median nerve trunk. A: position of electrode when wrist and fingers are extended. $B$ : the wrist and fingers are now flexed and the tip of the electrode is carried towards the shoulder in the long axis of the nerve.

abolished when the electrode was withdrawn by $1 \mathrm{~mm}$, indicating that adjacent soft tissues did not share in the movement. The distance moved by the free end of the electrode was measured with calipers to the nearest $0.5 \mathrm{~mm}$. The length of the electrode and its depth of insertion were also recorded, allowing the displacement of the tip to be calculated.

\section{RESLLTS}

The excursion of the tip of the needle, and thus of the trunk of the median nerve, depended upon the amount of limb movement. Active and passive movements had equal effects. Data from recordings in the upper arm are summarized in the Table. The greatest excursion of the electrode tip was produced by extension of the wrist and fingers $(7.4 \mathrm{~mm}$ downwards) and by flexion of the elbow (4.3 $\mathrm{mm}$ upwards). There were considerable differences between different subjects. In some the median nerve in the upper arm was displaced $2-3 \mathrm{~cm}$ in response to movement of the limb, and even a deep inspiration could draw the nerve towards the shoulder by as much as $8 \mathrm{~mm}$.

\section{T A B L E}

LONGITUDINAL SLIDING OF MEDIAN NERVE IN UPPER ARM IN RESPONSE TO MOVEMENTS OF LIMB, NECK, AND THORAX*

\begin{tabular}{|c|c|c|c|}
\hline \multirow[b]{2}{*}{ Movement } & \multicolumn{2}{|c|}{$\begin{array}{c}\text { Direction and amount of } \\
\text { nerve sliding }\end{array}$} & \multirow{2}{*}{$\begin{array}{c}\text { Subjects } \\
\text { in whom } \\
\text { no sliding } \\
\text { occurred } \\
\text { (no.) }\end{array}$} \\
\hline & $\operatorname{Mean}(\mathrm{m!m})$ & Range $(\mathrm{mm})$ & \\
\hline Flex fingers & $0.7 \uparrow$ & $0-3.2 \hat{\imath}$ & 4 \\
\hline Flex fingers and wrist & $1.6 ?$ & $0-6.8 \uparrow$ & 2 \\
\hline Extend fingers and wrist & $7.4 \downarrow$ & $2.8 \downarrow-20.5 \downarrow$ & 0 \\
\hline Pronation of forearm & $0.3 \uparrow$ & $2.2 \downarrow-1.0 \uparrow$ & 6 \\
\hline Supination of forearm & $0.9 \uparrow$ & $2.8-1.4 \uparrow$ & 5 \\
\hline Flexion of elbow to $45^{\circ}$ & 4.3 & $0-8.6 \uparrow$ & 1 \\
\hline Neck flexed forwards & $0.2 \uparrow$ & $2.8 \downarrow-2.2 \uparrow$ & 4 \\
\hline $\begin{array}{l}\text { Neck flexed towards nerve } \\
\text { Neck flexed away from }\end{array}$ & $0.4 !$ & $2.3 \hat{\imath}-5.1 \downarrow$ & 6 \\
\hline nerve & $2.8 \uparrow$ & $0-9.0 \uparrow$ & 2 \\
\hline $\begin{array}{l}\text { Neck rotated towards nerve } \\
\text { Neck rotated away from }\end{array}$ & $0.3 \uparrow$ & $3.0 \downarrow-5.6 \uparrow$ & 8 \\
\hline nerve & $1.1 \uparrow$ & $5.6 \downarrow-3.0 \uparrow$ & 6 \\
\hline Neck hyperextended & $1.2 \uparrow$ & $0.5 \downarrow-7.0 \uparrow$ & 8 \\
\hline Single deep inspiration & $4.5 \uparrow$ & $0-8.3 \uparrow$ & 1 \\
\hline
\end{tabular}

* Data from 15 subjects. $\uparrow=$ sliding towards the shoulder, $\downarrow=$ sliding towards the elbow.

In each of the four subjects in whom recordings were made at the wrist, upward displacement of 4-5 $\mathrm{mm}$ occurred when the index finger or middle finger was flexed. Extension of the wrist caused significant associated movement of the soft tissues through which the electrode passed, precluding accurate measurement of nerve displacement, but we estimated that hyperextension of the wrist caused the median nerve to slide downwards by $10-15 \mathrm{~mm}$ relative to bony landmarks at the lower end of the forearm. Displacement of the median nerve during flexion of the wrist and fingers was therefore two to four times greater at the wrist than in the upper arm. 


\section{DISCUSSION}

These observations suggest that longitudinal displacement is an important part of a peripheral nerve's accommodation to change in the length of its bed during movement of the limb. When the fingers flex, the median nerve slides upwards into the forearm and when the fingers extend it slides downwards towards the hand (Fig. 2). Movements of the wrist have the additional complication that the retinaculum tends to be carried proximally over the nerve during flexion. Extension of the fingers with flexion of the wrist is therefore the position in which the median nerve is displaced furthest under the retinaculum into the hand (Fig. 2). As the segment of nerve distal to the retinaculum is relatively short, providing little scope for stretch, the force of downward traction during finger extension is likely to be much greater than the upward traction, exerted only by the nerve's elasticity, during finger flexion.

Sunderland (1968) has studied the mechanical properties of peripheral nerves in formalin-
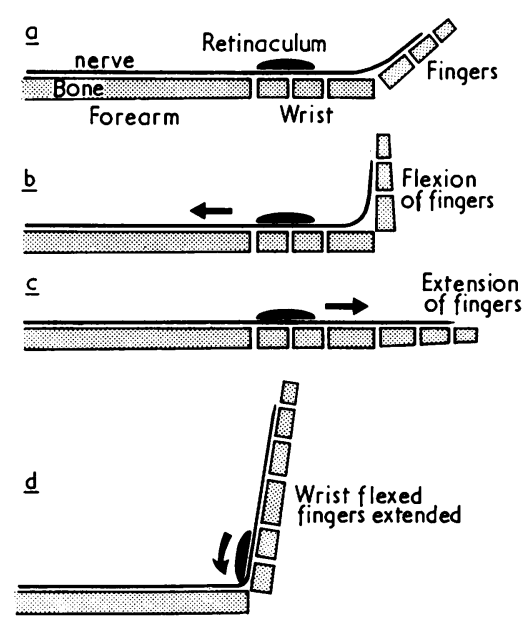

FIG. 2 Diagram illustrating relative displacement of the median nerve and flexor retinaculum at the wrist, starting at neutral position. (a) Semi-flexion of the fingers. (b) Nerve slides proximally when fingers flexed. (c) Nerve slides distally when fingers extended. (d) When fingers are kept extended while wrist is flexed, the retinaculum is carried proximally over the nerve trunk. fixed postmortem specimens. He found that the tensile strength of the nerve trunk depended essentially on the perineurium, the elastic properties of the nerve being retained so long as the perineurium remained intact. In his review of the effects of stretching upon nerve function, Sunderland (1968) concluded that a whole peripheral nerve could safely be stretched by $7 \%$ of its length without interfering with function, and that normal function could be preserved in some cases with a stretch of as much as $20 \%$.

Longitudinal sliding must obviate some of the local stretching that would otherwise be necessary during limb movement, for it allows unstretched lengths of nerve to slide into the extended region. Entrapment neuropathies are common in those who use their limbs for repetitive tasks over prolonged periods. This suggests that to-and-fro sliding through siteso of potential entrapment might cause foca $\bar{p}$ damage to a nerve.

The pathology of entrapment neuropathies is now well established. Visible swelling characteristically seen at the proximal edge of the trapped segment at the wrist or elbow Sometimes a smaller swelling is apparent at the distal edge also. It is not known why the swelling should be predominantly proximal rather than distal. Upton and McComas (1973) suggested that it could result from a damming-up of axonal flow but histological evidence suggests that the bulk of the visible swelling is caused by folding and thickening of the perineurium and not by swelling of the axons. Axonal degeneration may occur in severe entrapment neuropathies but in early uncomplicated entrapment neuropathy perineurial swelling and fibrosis may be found in the absence of detectable axonal degeneration (Weller, 1974). The myelinated fibres themselves show a characteristic distortion consisting of telescoping of myelin internodes with swelling and folding of the myelin sheath away from the entrapment in both directions (Ochoa and Marotte, 1973). Thus the myelin sheath of each segment is tapered at the end nearest the entrapment while a bulb of folded myelin is produced at the far end. Similar findings have been demonstrated at common sites of 
entrapment in nerves obtained at postmortem examination from subjects who had no symptoms during life (Neary et al., 1975). These authors have concluded that the histological changes are brought about by repeated pressure waves arising at the site of entrapment. If the point of reversal of polarity of the myelin deformities is taken to indicate the centre of the lesion, then in two of five median nerves the lesions were centred under the upper edge of the retinaculum and extended in both directions by $1-2 \mathrm{~cm}$. In the other three nerves the exact point of reversal could not be identified but the lesions were even longer, extending for 4-5 cm (Neary et al., 1975).

These studies have not explained why the perineurial swelling is greatest proximal to entrapment at the wrist. Observations of longitudinal sliding suggest that shearing forces deforming the nerve in a proximal direction are more severe than those deforming it towards the fingers. The position of the hand producing greatest proximal shearing would be one of extension of the fingers plus flexion of the wrist (Table; Fig. 2). Such forces could of course be accentuated by injury (especially posterior displacement) of the wrist or by the retinacular changes known to be associated with entrapment neuropathy.

One of the curious clinical features of entrapment syndromes is that symptoms may appear to arise proximal to the site of entrapment (Lishman and Russell, 1961; Crymble, 1968). Proximal symptoms do not occur after distal penetrating wounds or crush injuries and are therefore unlikely to be referred; conversely, referred pain arising from cervical lesions rarely involves the median nerve distribution in the hand (Hockaday and Whitty, 1967). Moreover, a close analysis of 146 patients with pain in the arm established that the occurrence of pain in the forearm is not influenced by the presence or absence of numness or paraesthesiae in the fingers (Lishman and Russell, 1961). Of these patients 74 found the symptoms were exacerbated by using the limb. Although the site of the symptoms was unhelpful in diagnosis, over half the patients could be diagnosed as having carpal tunnel syndromes on the basis of neurological ex- amination, while one-third of them had strong clinical or radiographic evidence of cervical spondylosis or cervical ribs. Upton and McComas (1973) reported that of 115 patients with carpal tunnel syndrome or ulnar entrapment at the elbow, 81 also had definite evidence of lesions of cervical nerve roots. In tracing the history of the clinical concept of brachial neuritis, Lishman and Russell (1961) pointed out that the accepted clinical features of the carpal tunnel syndrome are similar to those described by Walshe (1945) in lesions of the thoracic and axillary inlets, and to many reported examples of cervical rib compression and cervical spondylosis. Moreover, where genuine distal compression exists, all the symptoms may be relieved by local decompression, or by resting the whole limb. The authors suggested that pathological changes at one level-perhaps by altering the capacity for stretch-could make other levels vulnerable to damage during limb movement. Diffuse stretch-damage might thus be responsible for the widely dispersed symptoms, including the not uncommon finding of tenderness along much of the course of an affected nerve.

The fact that entrapment neuropathies are common in those who use their limbs for repetitive tasks over prolonged periods (Spaans, 1970) suggests that to-and-fro sliding across a site of potential entrapment might cause focal perineural damage leading to a restriction of sliding at that point. If sliding were restricted at more than one level, the capacity of the nerve to accommodate limb movement could be considerably reduced.

More histological information about the state of the nerve trunks in patients with entrapment neuropathy is required to test these hypotheses. Clearly it is unnecessary to propose external accidental trauma as a common cause for entrapment. We suggest that when longitudinal sliding of a peripheral nerve is restricted, continual and increasing damage results from normal movements of the limb.

We wish to thank the subjects for their co-cperation and The London Hospital, Ciba-Geigy Ltd., and the Multiple Sclerosis Society for financial support. 


\section{REFERENCES}

Clarke, E., and Bearn, J. G. (1972). The spiral nerve bands of Fontana. Brain, 95, 1-20.

Crymble, B. (1968). Brachial neuralgia and the carpal tunnel syndrome. British Medical Journal, 3, 470471.

Hockaday, J. M., and Whitty, C. W. M. (1967). Patterns of referred pain in the normal subject. Brain, 90, 481-496.

Lishman, W. A., and Russell, W. R. (1961). The brachial neuropathies. Lancet, 2, 941-947.

McLellan, D. L. (1975). Longitudinal sliding of median nerve during hand movements. Lancet, 1, 633-634.

Neary, D., Ochoa, J., and Gilliatt, R. W. (1975). Subclinical entrapment neuropathy in man. Journal of the Neurological Sciences, 24, 283-298.

Ochoa, J., and Marotte, L. (1973). The nature of the nerve lesion caused by chronic entrapment in the guinea-pig. Journal of the Neurological Sciences, 19, 491-495.
Spaans, F. (1970). Occupational nerve lesions. In Diseases of Nerves, part 1, vol. 7, of Handbook of Clinical Neurology, pp. 326-343. North-Holland: Amsterdam.

Sunderland, S. (1968). The mechanical properties of peripheral nerve trunks. In Nerves and Nerve Injuries, pp. 62-66. Livingstone: Edinburgh.

Upton, A. R. M., and McComas, A. J. (1973). The double crush in nerve entrapment syndromes. Lancet, 2, 359-362.

Vallbo, A. B., and Hagbarth, K-E. (1968). Activity from skin mechanoreceptors recorded percutaneously in awake human subjects. Experimental Neurology, 21, 270-289.

Walshe, F. M. R. (1945). On 'acroparaesthesia' and so-called 'neuritis' of the hands and arms in women. British Medical Journal, 2, 596-598.

Weller, R. (1974). 'Localised hypertrophic neuropathy' and hypertrophic polyneuropathy. Lancet, 2, 592-593. 